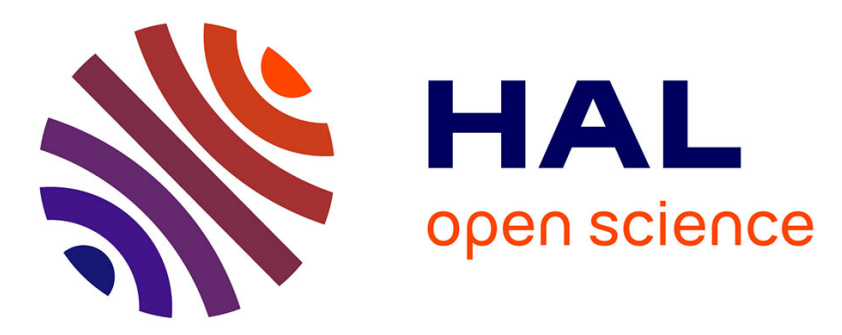

\title{
Integration Duration-Based Method for Unveiling Hidden Patterns of Even Number of Spirals of Chua Chaotic Attractor
}

\author{
Malika Belouerghi, Tidjani Menacer, René Lozi
}

\section{- To cite this version:}

Malika Belouerghi, Tidjani Menacer, René Lozi. Integration Duration-Based Method for Unveiling Hidden Patterns of Even Number of Spirals of Chua Chaotic Attractor. Indian Journal of Industrial and Applied Mathematics, 2019, 10 (1), pp.13-33. 10.5958/1945-919X.2019.00020.3 . hal-03044722

\author{
HAL Id: hal-03044722 \\ https://hal.science/hal-03044722
}

Submitted on 15 Dec 2020

HAL is a multi-disciplinary open access archive for the deposit and dissemination of scientific research documents, whether they are published or not. The documents may come from teaching and research institutions in France or abroad, or from public or private research centers.
L'archive ouverte pluridisciplinaire $\mathbf{H A L}$, est destinée au dépôt et à la diffusion de documents scientifiques de niveau recherche, publiés ou non, émanant des établissements d'enseignement et de recherche français ou étrangers, des laboratoires publics ou privés. 


\title{
Integration Duration-Based Method for Unveiling Hidden Patterns of Even Number of Spirals of Chua Chaotic Attractor
}

\author{
Malika Belouerghi ${ }^{1}$, Tidjani Menacer ${ }^{2}$ and René Lozi $^{3 *}$ \\ ${ }^{1,2}$ Department of Mathematics, University Mohamed Khider, Biskra, Algeria. \\ ${ }^{3}$ University Côte d'Azur, CNRS, LJAD, Parc Valrose 06108, Nice Cédex 02, France. \\ (*Corresponding author) E-mail: *Rene.LOZI@univ-cotedazur.fr \\ ${ }^{1}$ belouerghimalika@yahoo.fr, ${ }^{2}$ tidjanimenacer@yahoo.fr
}

\begin{abstract}
The purpose of this article is to introduce a novel method for unveiling hidden patterns of even number of spirals in the multi-spiral Chua Chaotic attractor. This method is based on the duration of integration of this system and allows displaying every pattern containing from 1 to $c+1$ spirals of this attractor. After having given the equation of the multispiral Chua Chaotic attractor, the Menacer-Lozi-Chua (MLC) method for uncovering hidden bifurcations is explained again and a numerical example of a route of bifurcation is given. In the chosen example, the attractors found along this hidden bifurcation route display an odd number of spirals. With the novel method, during the integration process, before reaching the asymptotical attractor which possesses an odd the number of spirals, the number of spirals increases one by one until it reaches the maximum number corresponding to the value fixed by $\varepsilon$, unveiling both patterns of even and odd number of spirals.
\end{abstract}

Keywords: hidden bifurcation, Chua's circuit, pattern of even number of spirals

\section{INTRODUCTION}

The name 'bifurcation' was first introduced by Henri Poincaré in $1885^{[1]}$ in the frame of the qualitative theory of dynamical systems. A bifurcation occurs when, for a fixed value of a parameter, the number of solutions of a family of differential equations is increased from one to two, like the bifurcation of a branch of a tree ${ }^{[2,3]}$, or when the topological nature of the solution changes from steady state to periodic function (Hopf bifurcation ${ }^{[4]}$ ).

In 1963, the discovery of the first chaotic strange attractor by Lorenz ${ }^{[5]}$ has broadened the field of the bifurcation theory. The first system of differential equation modelling real electronic device for which the asymptotic attractor is chaotic (hence a strange attractor) was invented by $\mathrm{Chua}^{[6]}$ during a stay in the laboratory of professor Matsumoto, at Waseda University, Tokyo.

Chua attractor is nowadays incredibly used because both of its realisations: electronic circuit or system of differential equations can be combined for multiple purposes ${ }^{[7]}$. Chua circuit is the simplest electronic circuit exhibiting chaos and possesses a very rich dynamical 
behaviour which was verified from numerous laboratory experiments ${ }^{[8]}$, computer $^{2}$ simulations $^{[9]}$ and rigorous mathematical analysis ${ }^{[10-12]}$.

Since that discovery, the Chua circuit has been generalised to several versions. One generalisation substitutes the continuous piecewise linear function by a smooth function, such as a cubic polynomial ${ }^{[13-16]}$, etc. Complex attractors with $n$-double spirals were reported in Suykens and Vandewalle ${ }^{[17]}$ by introducing additional break points in the non-linear element in the Chua's circuit or using cellular neural networks with a piecewise linear output function $^{[18]}$.

It is also demonstrated in Suykens et al. and Yalçin et al. ${ }^{[19,20]}$; odd number of scrolls can be observed by a similar modification. In Tang et al. $\left.{ }^{[21}\right]$, it is shown that the $n$-spirals attractors can be obtained with a simple sine or a cosine function.

In 2011, Leonov et al. introduced a new classification of attractors of dynamical systems with the concept of hidden attractor and self-excited attractor ${ }^{[22,23]}$. They showed that the basin of attraction of a self-excited attractor overlaps with the neighbourhood of an equilibrium point; therefore, these attractors are very easy to be found, because it suffices to give the equilibrium point as an initial condition, if this equilibrium point is unstable. On the contrary, a hidden attractor has a basin of attraction that does not intersect with small neighbourhoods of any equilibrium points, thereby making it very difficult to find. The name hidden comes from this property.

Hidden attractors are important in engineering applications because they allow unexpected and potentially disastrous responses to perturbations in a structure like aircrafts control systems (windup and anti-windup) and electrical machines. An effective method for the numerical localisation of hidden attractors in multi-dimensional dynamical systems has been proposed by these Leonov and Leonov et al. ${ }^{[24,25]}$. This method is based on homotopy and numerical continuation. They construct a sequence of similar systems such that for the first (starting) system the initial condition for numerical computation of oscillating solution (starting oscillation) can be obtained analytically. Then the transformation of this starting solution is tracked numerically in passing from one system to another. The first example of a hidden chaotic strange attractor was found in the Chua attractor ${ }^{[26]}$.

In their study of the modified multi-spiral Chua system with sine function ${ }^{[21]}$, Menacer et $a l .{ }^{[27]}$, using the Kuznetsov and Leonov method in another way, found a sequence of hidden bifurcations.

In this system, the parameter $c$ governing the number of spirals is an integer; hence, it is not possible to vary it continuously and therefore it is not possible to observe bifurcations of attractors from $n$ to $n+2$ spirals when the parameter $c$ changes. Moreover, it is not possible to use non-integer real values for $c$. To overcome this obstacle, they introduced a new bifurcation parameter $\varepsilon$ provided by the method of researching hidden attractor.

Next, in 'Multiple-spiral attractors in Chua's sine function system' section, the multispiral Chua system with sine function is recalled. In 'The Menacer-Lozi-Chua (MLC) method for method for uncovering hidden bifurcations' section, the Menacer-Lozi-Chua (MLC) method for uncovering hidden bifurcations is explained again. In the chosen example, it shown that the attractors along the hidden bifurcation route display an odd number of spirals. In 'Integration duration method for unveiling hidden patterns of even number of spirals' section, we introduce a novel method for unveiling hidden patterns of even number of 
spirals. This method based on the duration of integration of the considered system. During the integration process, before reaching the asymptotical attractor which possesses and odd the number of spirals, the number of spirals increases until it reaches the maximum number corresponding to the value fixed by $\varepsilon$. Finally, in 'Conclusion' section, a brief conclusion is drawn.

\section{MULTIPLE-SPIRAL ATTRACTORS IN CHUA'S SINE FUNCTION SYSTEM}

Complex chaotic attractors with $n$-double scrolls using cellular neural networks with a piecewise linear output function were reported in Arena et al. ${ }^{[18]}$. In Tang et al. ${ }^{[21]}$, a new family of continuous functions for generating $n$-scroll attractors is proposed. It is shown that $n$-scroll attractors can be obtained with a simple sine or cosine function. Since then in the last decades, multi-scroll chaotic attractors generation has been extensively studied due to their promising applications in various real-word chaos-based technologies including secure and digital communications, random bit generation and so on.

The system of differential equations, describing the behaviour of Chua's circuits, is threedimensional with a piecewise linear non-linearity ${ }^{[6,28]}$.

$$
\left\{\begin{array}{c}
\dot{x}(t)=\alpha(y(t)-f(x(t))) \\
\dot{y}(t)=x(t)-y(t)-z(t) \\
\dot{z}(t)=-\beta y(t)
\end{array}\right.
$$

with $\dot{x}(t)=\frac{d x(t)}{d t}, \quad \dot{y}(t)=\frac{d y(t)}{d t}, \quad \dot{z}(t)=\frac{d z(t)}{d t}$

For the Chua's modified system with a sine function, the non-linearity reads (Figure 1):

$$
f(x(t))=\left\{\begin{array}{ccc}
\frac{b \pi}{2 a}(x(t)-2 a c), & \text { if } & x(t) \geq 2 a c \\
-b \sin \left(\frac{\pi x(t)}{2 a}+d\right), & \text { if } & -2 a c \leq x(t) \leq 2 a c \\
\frac{b \pi}{2 a}(x(t)+2 a c), & \text { if } & x(t) \leq-2 a c
\end{array}\right.
$$

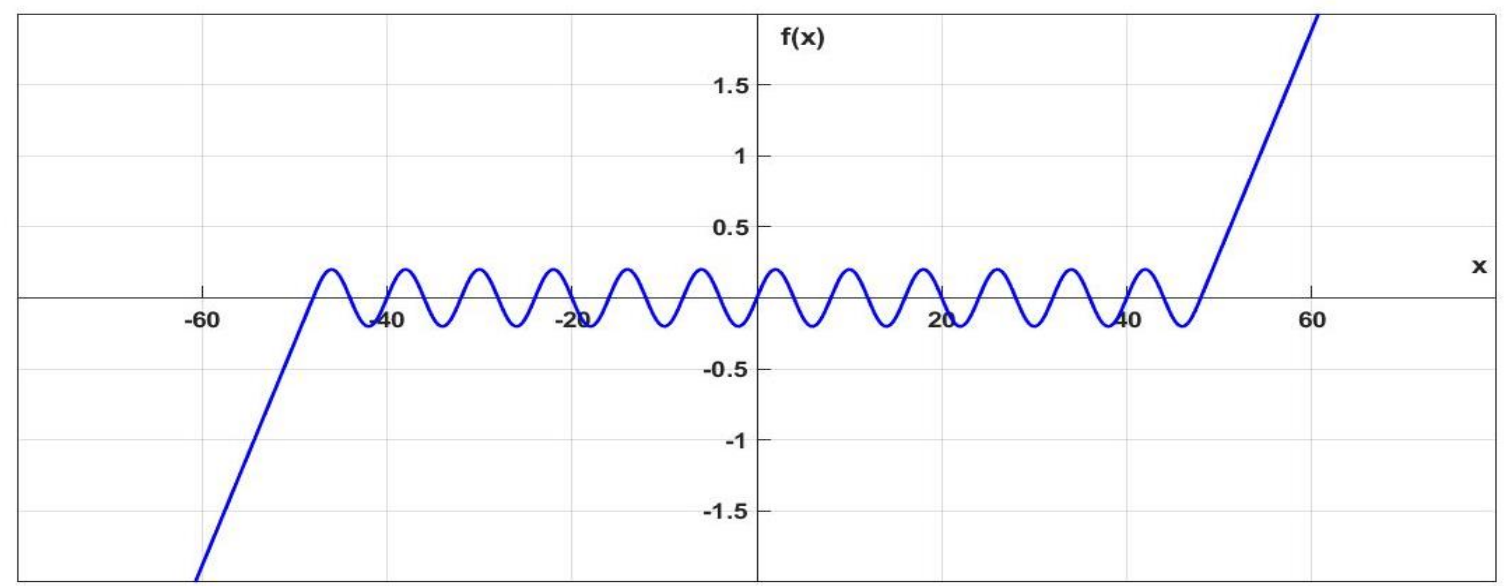

Figure 1. Proposed Sine Function $f(x)$ with Parameters Values $a=2, b=0.2, c=12, d=\pi$. 
In this article, rather than considering the fractal structure of the attractors, we highlight their global geometric feature, that is, we only consider their general shape described in terms of the number of spirals. We fix the real parameter values $\alpha=11, \quad \beta=15, \quad a=2, \quad b=$ 0.2 for which one can find chaotic attractors topologically equivalent to the attractors described in Ref. [21].

The parameter $c$ is an integer which governs the number $n$ of spirals.

$$
n=c+1
$$

and $d$ is chosen such that

$$
d=\left\{\begin{array}{lllll}
\pi & \text { if } & c & \text { is } & \text { even } \\
0 & \text { if } & c & \text { is } & \text { odd }
\end{array}\right.
$$

A straightforward computation gives the equilibrium points which are $\left(-x_{e q}, 0, x_{e q}\right)$ with $x_{e q}=2 a k$ and $k=0, \pm 1, \pm 2, \ldots, \pm c[2]$.

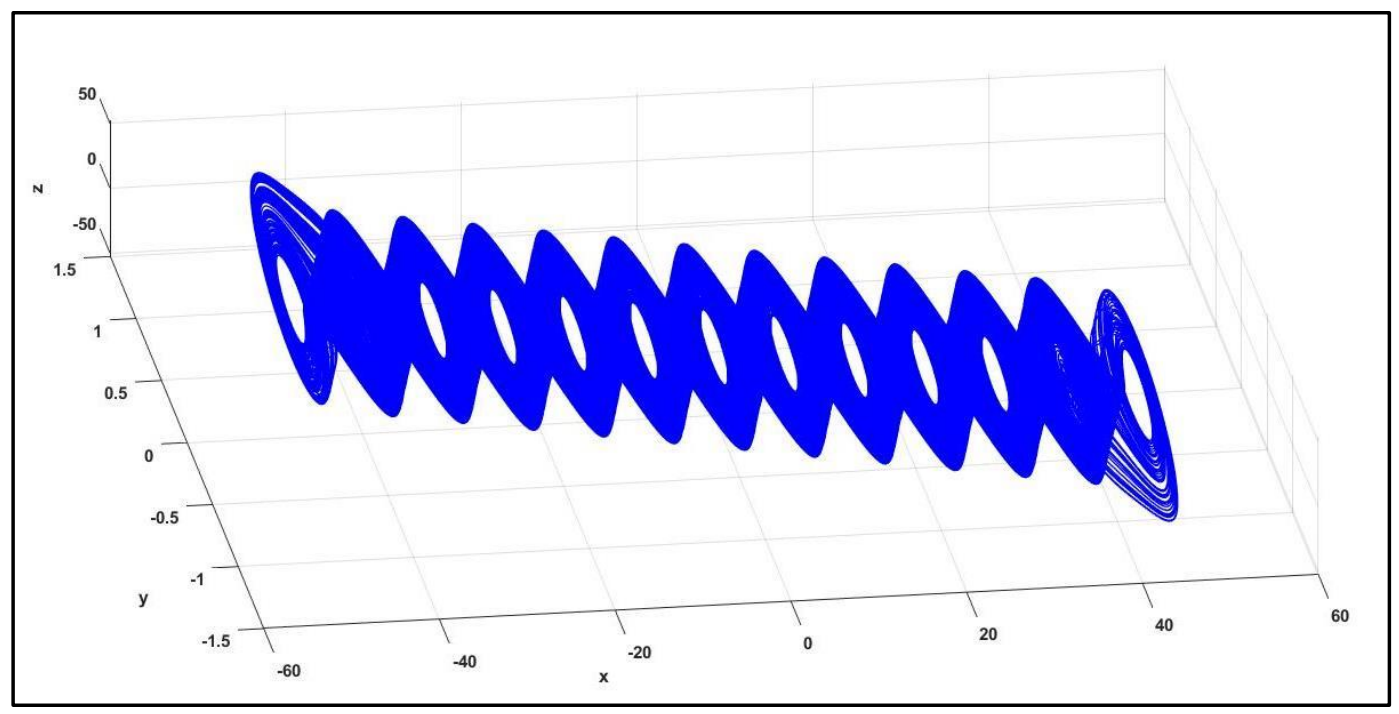

(a)

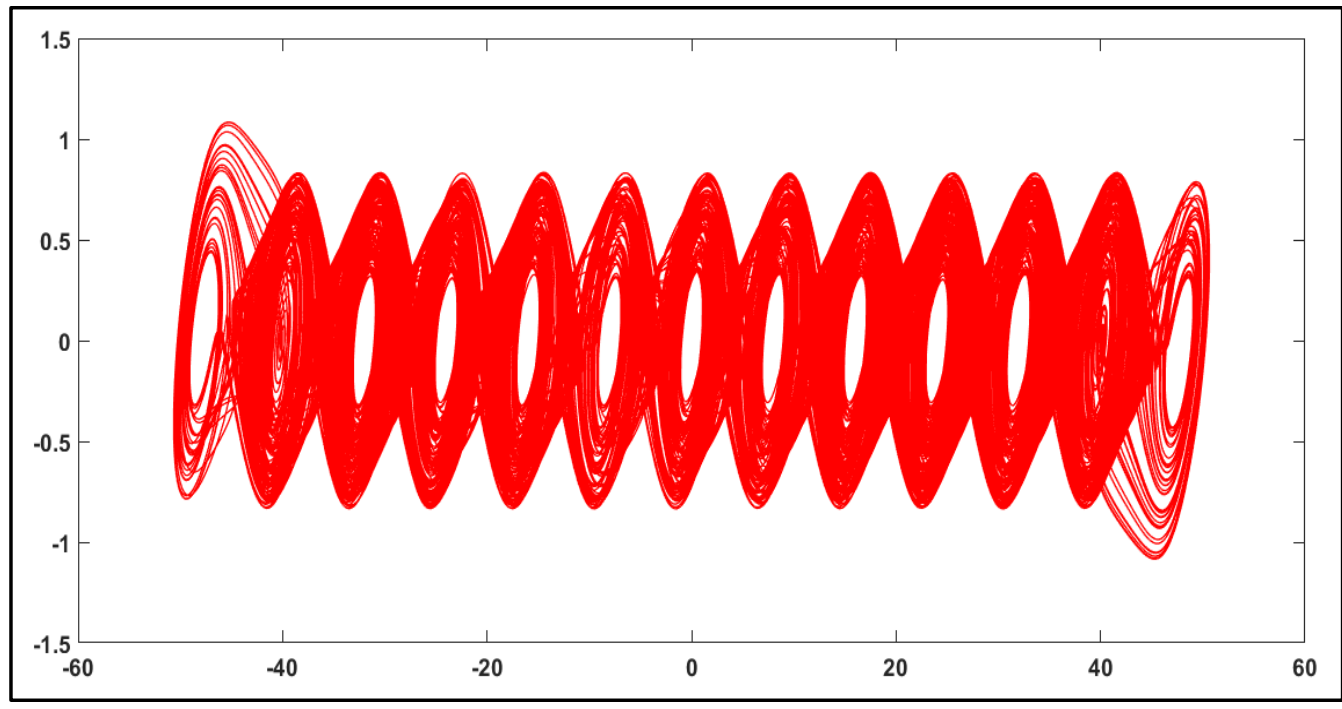

(b)

Figure 2. The 13-Spiral Attractor Generated by Equations (1) and (2) for $c=12$. (a) 3-Dimensional Figure, (b) Projection onto the Plane (x, y). 
In order to compare the new integration duration based method versus the method previously introduced by Menacer et $a l .{ }^{[27]}$ we need a benchmark sequence of hidden bifurcations. In this goal, we limit our study to the case where parameter $c$ is set to the value 12 which gives a 13 spirals attractor (Figure 2). This number of scrolls is enough to obtain a significant comparison. For a complete study of this model, with other values of $c$ see Ref. [27].

\section{THE MLC METHOD FOR METHOD FOR UNCOVERING HIDDEN BIFURCATIONS}

In the modified Chua circuit with sine function (1)-(2), the parameter $c$ governing the number of spirals is an integer; hence, it is not possible to vary it continuously and therefore it is not possible to observe bifurcations of attractors from $n$ to $n+2$ spirals when the parameter $c$ changes. Moreover, it is not possible to use non-integer real values for $c$. To overcome this obstacle, [27] introduced a new method for uncovering hidden bifurcations based on the core idea of the Leonov and Kuznetsov for searching hidden attractors (i.e. homotopy and numerical continuation, see Appendix). While keeping $c$ constant, a new bifurcation parameter $\varepsilon$ is introduced. We recall briefly this method in this section in which the value of parameters is fixed at $\alpha=11, \quad \beta=15, \quad a=2, \quad b=0.2, \quad c=12, \quad d=\pi$.

\section{The Method}

For this purpose, we rewrite System (1)-(2) in the form Lur'e's form [29]

$$
\frac{d X}{d t}=M X+q H\left(r^{T} X\right) \quad X \in R^{3},
$$

where $M=\left(\begin{array}{ccc}0 & \alpha & 0 \\ 1 & -1 & 1 \\ 0 & -\beta & 0\end{array}\right), \quad X=\left(\begin{array}{l}x \\ y \\ z\end{array}\right), \quad q=\left(\begin{array}{c}-\alpha \\ 0 \\ 0\end{array}\right), \quad r=\left(\begin{array}{l}1 \\ 0 \\ 0\end{array}\right)$, and $H(\sigma)=f(\sigma)$.

We introduce a coefficient $\chi$ and a small parameter $\varepsilon$ in order to transform System (5) into the form similar to the system (V) as follows

$$
\frac{d X}{d t}=M_{0} X+q \varepsilon g^{0}\left(r^{T} X\right), \quad X \in R^{3}
$$

where

$$
M_{0}=M+\chi q r^{T}=\left(\begin{array}{ccc}
-\alpha \chi & \alpha & 0 \\
1 & -1 & 1 \\
0 & -\beta & 0
\end{array}\right)
$$

and

$$
g(\sigma)=H(\sigma)-\chi \sigma=-\alpha f(\sigma)-\chi \sigma .
$$

Then considering the transfer function $W(m)(\mathrm{XI})$ solving the equations $\operatorname{Im} W\left(i \omega_{0}\right)=0$ and $\chi=-\operatorname{Re} W\left(i \omega_{0}\right)^{-1}$, which obtains $\omega_{0}=2.1018$ and $\chi=0.03796$. 
By the non-singular linear transformation, $X=S Z$ the System (6) is reduced to the form similar to Equation (VI)

$$
\frac{d Z}{d t}=A Z+B \varepsilon g^{0}\left(C^{T} Z\right), \quad Z \in R^{3}
$$

where

$$
A=\left(\begin{array}{ccc}
0 & -\omega_{0} & 0 \\
\omega_{0} & 0 & 0 \\
0 & 0 & -d_{1}
\end{array}\right), \quad Z=\left(\begin{array}{c}
z_{1} \\
z_{2} \\
z_{3}
\end{array}\right), \quad B=\left(\begin{array}{c}
b_{1} \\
b_{2} \\
1
\end{array}\right), \quad C=\left(\begin{array}{c}
1 \\
0 \\
-h
\end{array}\right) .
$$

Remark: The System (1) being 3-dimensional, $Z_{3}$ of Equation (VI) is a scalar denoted here $z_{3}$

This implies that

$$
A=S^{-1} M_{0} S, \quad B=S^{-1} q, \quad C^{T}=r^{T} S, \text { and } S=\left(\begin{array}{lll}
s_{11} & s_{12} & s_{13} \\
s_{21} & s_{22} & s_{23} \\
s_{31} & s_{32} & s_{33}
\end{array}\right)
$$

The transfer function $W_{A}(m)$ of System (7) reads

$$
W_{A}(m)=\frac{-b_{1} m+b_{2} \omega_{0}}{m^{2}+\omega_{0}^{2}}+\frac{h}{m+d_{1}} .
$$

Then, using the equality of transfer of both functions $W_{A}(m)$ and $W_{M_{0}}(m)=r^{T}\left(M_{0}-m I\right)^{-1} q$ of Systems (6) and (7), we obtain the following relations

$$
\begin{gathered}
\chi=\frac{a+\omega_{0}^{2}-\beta}{\alpha}, \\
d_{1}=\alpha+\omega_{0}^{2}-\beta+1, \\
h=\frac{\alpha\left(\beta-d_{1}+d_{1}^{2}\right)}{\omega_{0}^{2}+d_{1}^{2}}, \\
b_{1}=\frac{\alpha\left(\beta-\omega_{0}^{2}-d_{1}\right)}{\omega_{0}^{2}+d_{1}^{2}}, \\
b_{2}=\frac{\alpha\left(1-d_{1}\right)\left(\omega_{0}-\beta d_{1}\right)}{\omega_{0}^{2}\left(\omega_{0}^{2}+d_{1}^{2}\right)} .
\end{gathered}
$$

Numerical Example of Hidden Bifurcation Route

For the value of parameters fixed in this section, one obtains

$$
\chi=0.03796, \quad d_{1}=1.4176, \quad h=26.686, \quad b_{1}=15.686, \quad b_{2}=3.1003,
$$

And solving the matrix Equation (9) one gets $S=\left(\begin{array}{ccc}1 & 0 & -26.686 \\ 0.03795 & -0.19107 & -2.4264 \\ -1.3636 & -0.27084 & -25.674\end{array}\right)$.

Using Theorem 1 in the Appendix, for $\varepsilon$ small enough, one obtains the following initial condition for the first step of the multi-stage localisation procedure: 


$$
X^{0}(0)=S Z(0)=S\left(\begin{array}{c}
\tau_{0} \\
0 \\
0
\end{array}\right)=\left(\begin{array}{c}
\tau_{0} S_{11} \\
\tau_{0} S_{21} \\
\tau_{0} S_{31}
\end{array}\right)
$$

Let us apply the localisation procedure described in the Appendix. First, we compute the starting frequency $\omega_{0}$ and the coefficient of harmonic linearisation $\chi$ (already computed in 'The method' section). Using Equation (10), we obtain the initial conditions for the first step.

Then, we compute the solutions of System (6) with the non-linearity $\varepsilon g(x)=$ $\varepsilon(H(x)-\chi x)$, by increasing sequentially $\varepsilon$ from the value $\varepsilon=0.1$ to $\varepsilon=1$ by starting at the beginning with the Step 0.1 and decreasing progressively it, down to 0.001 between $\varepsilon=0.8$ and $\varepsilon=1$.

If the stable periodic solution $X^{1}(t)$ (corresponding to the small value $\varepsilon=0.1$ near the harmonic one is determined, all the points of this stable periodic solution are located in the domain of attraction of the stable periodic solution $X^{2}(t)$ of the system corresponding to $\varepsilon=$ 0.2 ). The solution $X^{2}(t)$ can be found numerically by searching one trajectory of System (5) with $\varepsilon=0.2$ taking as an initial point $X^{1}\left(t_{\max }\right)$ where $t_{\max }$ is the last value of the integration time. For the value of the parameter $\varepsilon=0.2$ after a transient regime, the computational procedure reaches the periodic solution $X^{2}(t)$. We continue by increasing the parameter $\varepsilon$ using the same numerical procedure to obtain $X^{3}(t), X^{4}(t), \cdots, X^{j}(t), \cdots$ which are solutions of Systems (1)-(2) for peculiar initial conditions. When $\varepsilon=0.8$, the first chaotic solution possessing one scroll is found.

We apply the above procedure to obtain the initial conditions for the solutions for increasing values of $\varepsilon$ as shown in Table 1 .

Table 1. Initial Condition According to the Values of $\varepsilon$

\begin{tabular}{|c||c||c|c|c||}
\hline $\boldsymbol{\varepsilon}$ & $\boldsymbol{X}^{\boldsymbol{j}}(\mathbf{0})$ & $\boldsymbol{x}_{\mathbf{0}}$ & $\boldsymbol{y}_{\mathbf{0}}$ & $\boldsymbol{z}_{\mathbf{0}}$ \\
\hline \hline 0.1 & $X^{1}(0)=X^{0}\left(t_{\max }\right)$ & 74.72 & 2.8356 & -101.89 \\
\hline 0.2 & $X^{2}(0)=X^{1}\left(t_{\max }\right)$ & -3.5603 & 0.0978 & 5.1616 \\
\hline 0.3 & $X^{3}(0)=X^{2}\left(t_{\max }\right)$ & 3.7396 & 0.1166 & -5.1258 \\
\hline 0.4 & $X^{4}(0)=X^{3}\left(t_{\max }\right)$ & 1.6312 & 0.7179 & -1.3015 \\
\hline 0.5 & $X^{5}(0)=X^{4}\left(t_{\max }\right)$ & 2.9797 & 0.5334 & -3.5896 \\
\hline 0.6 & $X^{6}(0)=X^{5}\left(t_{\max }\right)$ & 3.1137 & -0.2868 & -4.7176 \\
\hline 0.7 & $X^{7}(0)=X^{6}\left(t_{\max }\right)$ & -3.2894 & -0.5358 & 3.9445 \\
\hline 0.8 & $X^{8}(0)=X^{7}\left(t_{\max }\right)$ & -3.5390 & 0.2601 & 4.9005 \\
\hline 0.86 & $X^{9}(0)=X^{8}\left(t_{\max }\right)$ & -3.5920 & 0.1733 & 5.0637 \\
\hline 0.9785 & $X^{10}(0)=X^{9}\left(t_{\max }\right)$ & 10.5751 & 0.1758 & -11.8656 \\
\hline 0.989 & $X^{11}(0)=X^{10}\left(t_{\max }\right)$ & -12.1911 & 0.3828 & 11.2562 \\
\hline 0.993 & $X^{12}(0)=X^{11}\left(t_{\max }\right)$ & -7.4755 & -0.6595 & 5.6561 \\
\hline 0.9953 & $X^{13}(0)=X^{12}\left(t_{\max }\right)$ & 8.9249 & 0.7895 & -7.6282 \\
\hline 0.9994 & $X^{14}(0)=X^{13}\left(t_{\max }\right)$ & -2.8006 & -0.2619 & 3.9268 \\
\hline 1 & $X^{15}(0)=X^{14}\left(t_{\max }\right)$ & -2.6705 & 0.2845 & 2.2206 \\
\hline
\end{tabular}


Using these initial conditions, we get the solutions $X^{8}(t)$ (Figure 3a) with one scroll to $X^{14}(t)$ (Figure $5 \mathrm{~g}$ ). In each figure, there are a different odd number of spirals in the attractor. The number of spiral increases by 2 at each step as displayed in Table 2 from 1 to 13 spirals. The values of $\varepsilon$ in this table are exactly the values of bifurcation points. In Figure 3, we display the hidden bifurcations of the multi-spiral Chua's attractor.

Table 2. Values of Parameter $\varepsilon$ at the Bifurcation Points for $c=12$ (13 Spirals)

\begin{tabular}{|c|c|c|c|c|c|c|}
\hline \multicolumn{7}{|c|}{$\varepsilon$} \\
\hline 0.80 & 0.86 & 0.9785 & 0.989 & 0.993 & 0.9953 & 0.9994 \\
\hline$X^{8}(0)$ & $X^{9}(0)$ & $X^{10}(0)$ & $X^{11}(0)$ & $X^{12}(0)$ & $X^{13}(0)$ & $X^{14}(0)$ \\
\hline 1 spiral & 3 spirals & 5 spirals & 7 spirals & 9 spirals & 11 spirals & 13 spirals \\
\hline
\end{tabular}

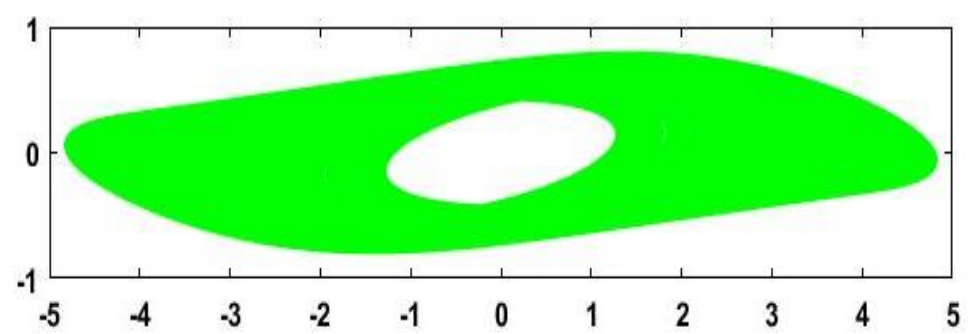

(a)

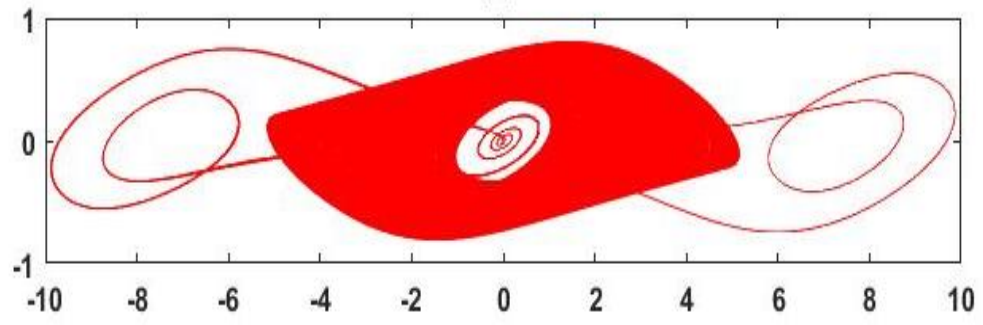

(b)

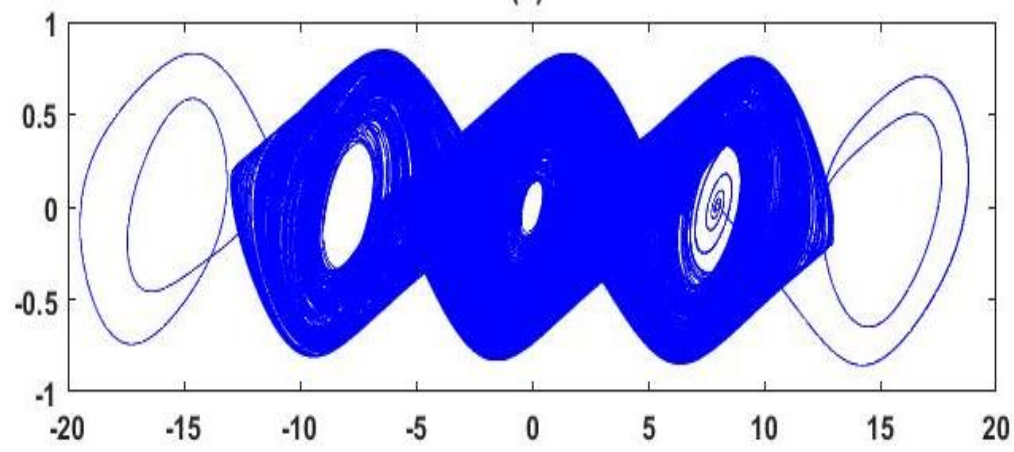

(c)

Figure 3. (Continued) 

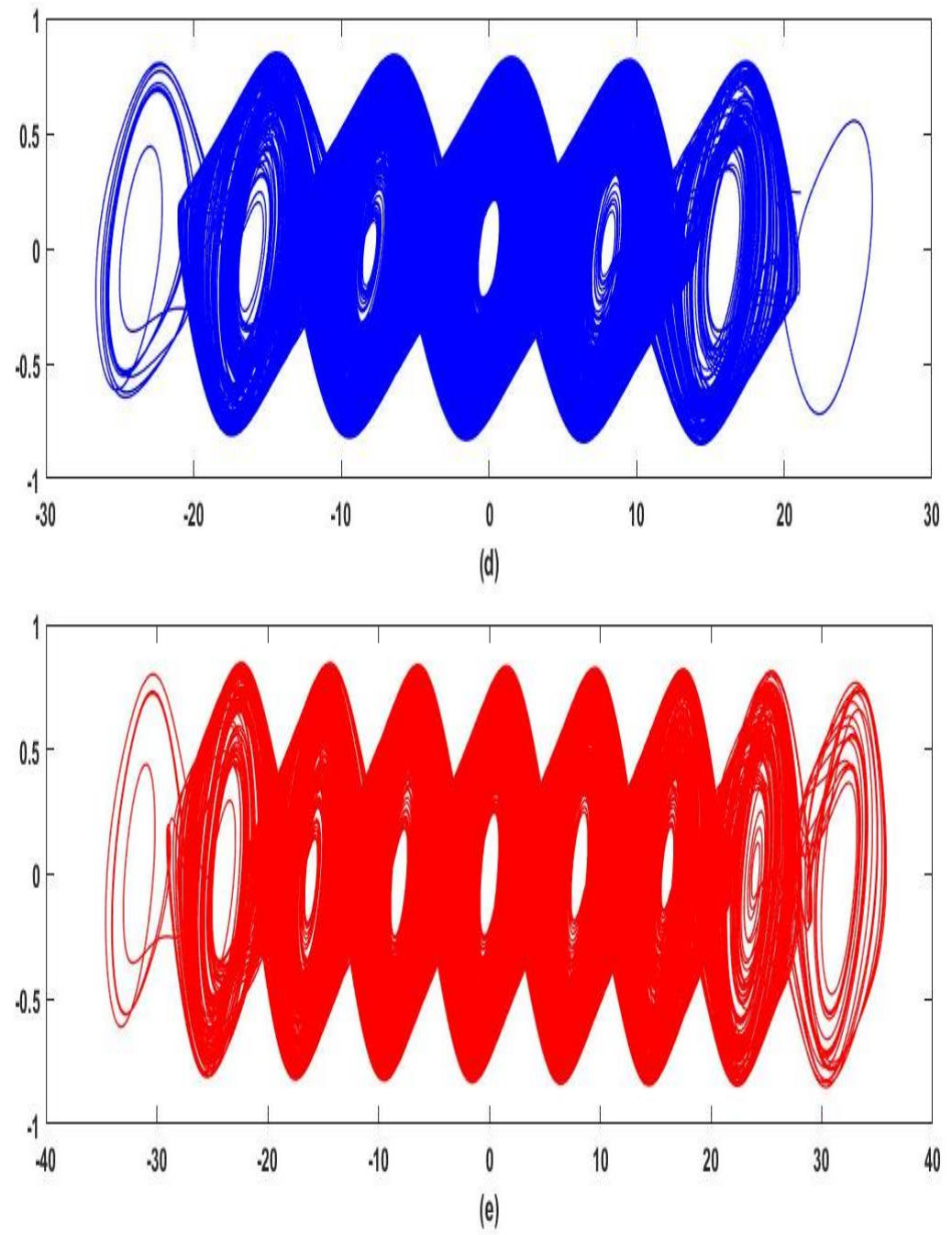

Figure 3. (Continued) 

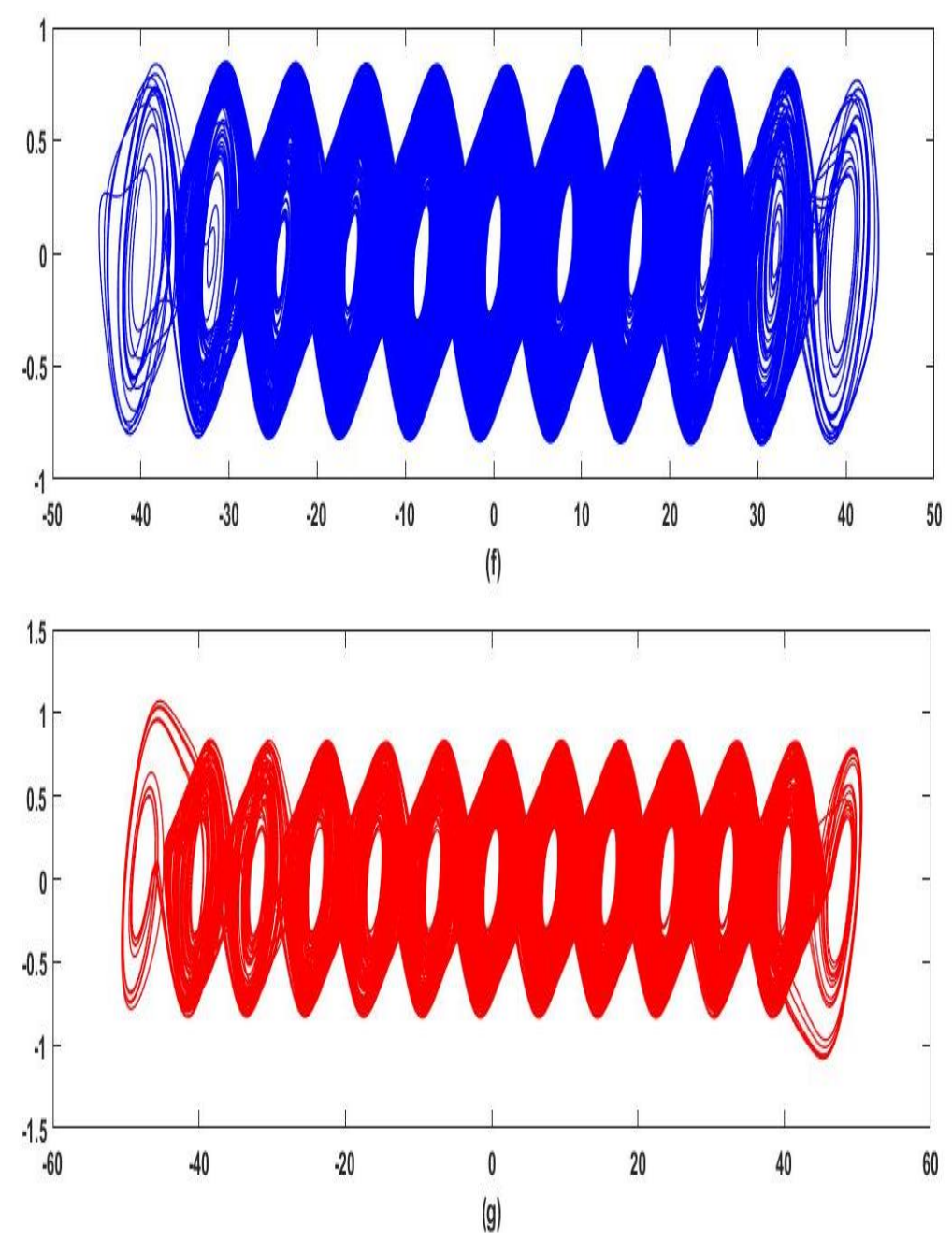

Figure 3. The Increasing Number of Spirals of System (6) According to Increasing $\varepsilon$ Values. (a) 1 -Spiral for $\varepsilon=$ 0.80, (b) 3-Spirals for $\varepsilon=0.86$, (c) 5-Spirals for $\varepsilon=0.9785$, (d) 7-Spirals for $\varepsilon=0.989$, (e) 9-Spirals for $\varepsilon=0.993$, (f) 11-Spirals for $\varepsilon=0.9953,(\mathrm{~g}) 13$-Spirals for $\varepsilon=0.9994$

\section{INTEGRATION DURATION METHOD FOR UNVEILING HIDDEN PATTERNS OF EVEN NUMBER OF SPIRALS}

In the previous section, it is shown that the attractors along the hidden bifurcation route display an odd number of spirals. In this section, we introduce a novel method for unveiling hidden patterns of even number of spirals. This method based on the duration of integration of Systems (1)-(2). For this novel method, we fix $\varepsilon$ and we increase the duration of integration $t_{\max }$. During the integration process, before reaching the asymptotical attractor which possesses and odd the number of spirals, the number of spirals increases until it reaches the maximum number corresponding to the value fixed by $\varepsilon$.

As a numerical example, we set the value of $\varepsilon$ to 0.9953 . For this value, 11 spirals belong to the asymptotic attractor (Figure3f). All the patterns are displayed in Figure 4 and the value of $t_{\text {stepmax }}$ is given in Table 3 . In each figure, the number of spiral increases by 1 (instead of 2 in the MLC method). The MATLAB's standard solver for ordinary differential equations ode45 is used in order to integrate the Chua's system. This function implements a Runge- 
Kutta method with a variable time step. Therefore, it is difficult to know $t_{\max }$. The value $t_{\text {stepmax }}$ (shown in Table 3) which is the maximum number of steps used (i.e. corresponding to the duration of integration).

Table 3. Values of $t_{\text {stepmax }}$ for $\varepsilon=0.9953$ and $c=12$

\begin{tabular}{|l|l|l|l|l|l|l|}
\hline $\boldsymbol{t}_{\text {stepmax }}$ & \multicolumn{1}{|c|}{$\mathbf{1 4 4}$} & $\mathbf{2 0 5 0}$ & $\mathbf{2 0 7 0}$ & $\mathbf{2 7 0 0}$ & $\mathbf{6 0 9 0}$ & $\mathbf{6 7 0 0}$ \\
\hline Number of spirals & 1 & 2 & 3 & 4 & 5 & 6 \\
\hline Figure & $4 \mathrm{a}$ & $4 \mathrm{~b}$ & $4 \mathrm{c}$ & $4 \mathrm{~d}$ & $4 \mathrm{e}$ & $4 \mathrm{f}$ \\
\hline $\boldsymbol{t}_{\text {stepmax }}$ & 9590 & 14,000 & 70,000 & 88,210 & 200,000 & \\
\hline Number of spirals & 7 & 8 & 9 & 10 & 11 & \\
\hline Figure & $4 \mathrm{~g}$ & $4 \mathrm{~h}$ & $4 \mathrm{i}$ & $4 \mathrm{j}$ & $4 \mathrm{k}$ & \\
\hline
\end{tabular}

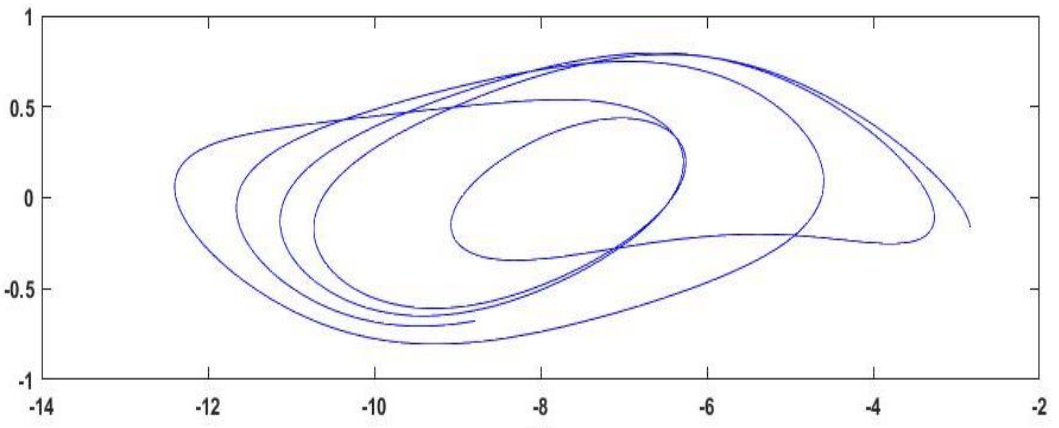

(a)

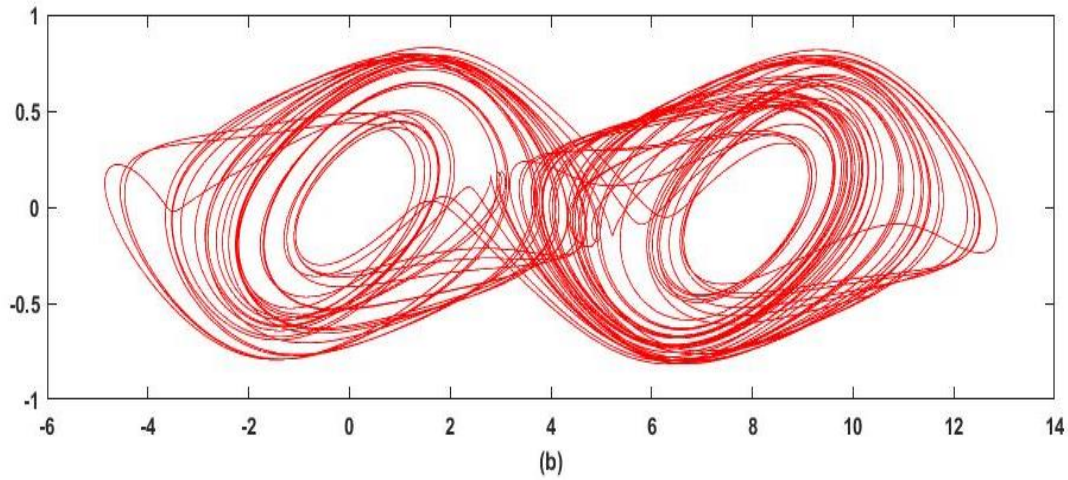

Figure 4. (Continued) 


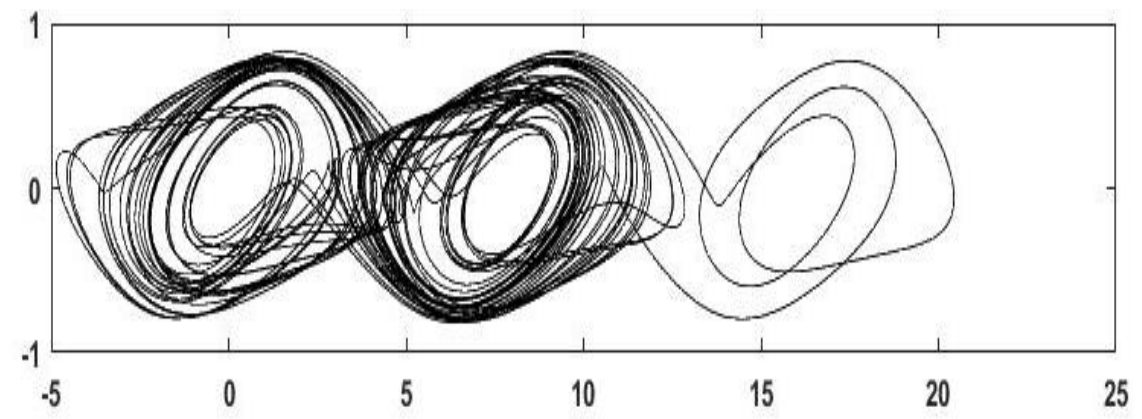

(c)

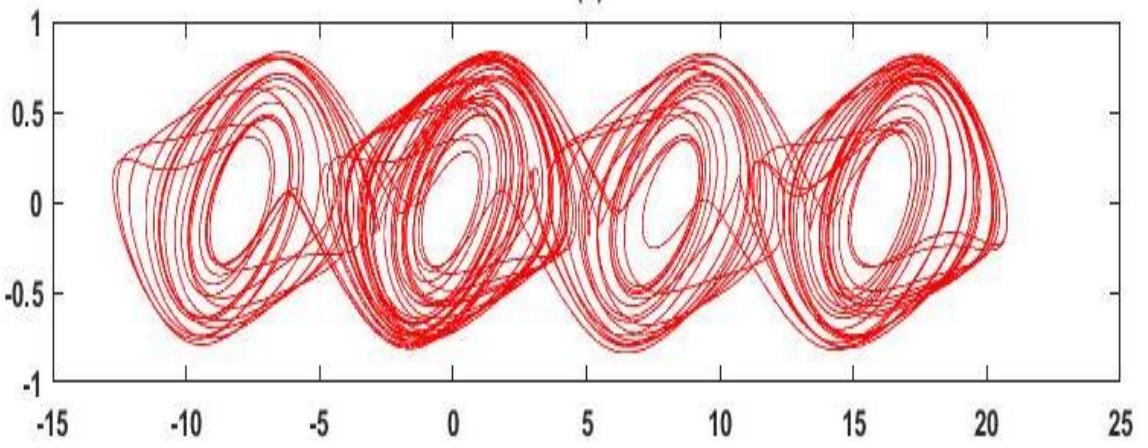

(d)

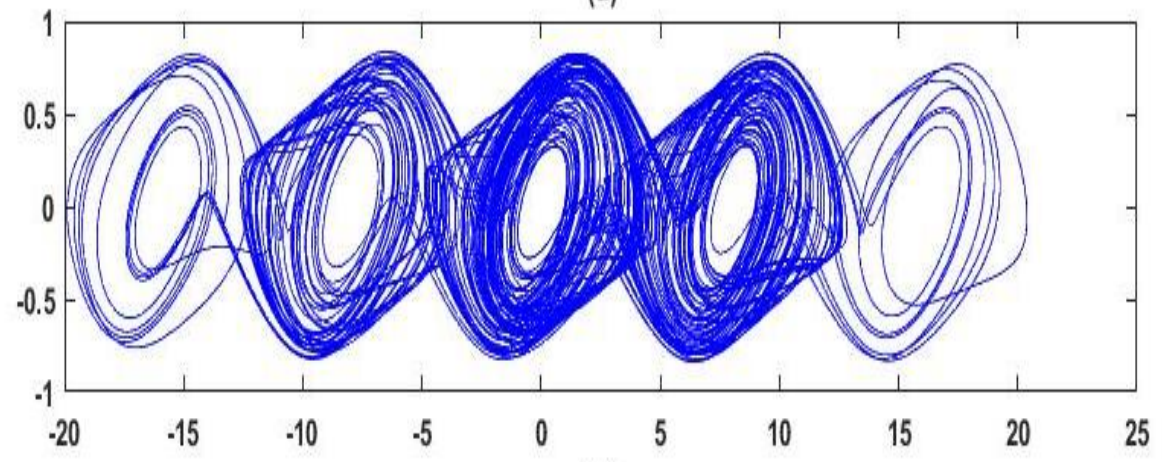

(e)

Figure 4. (Continued) 

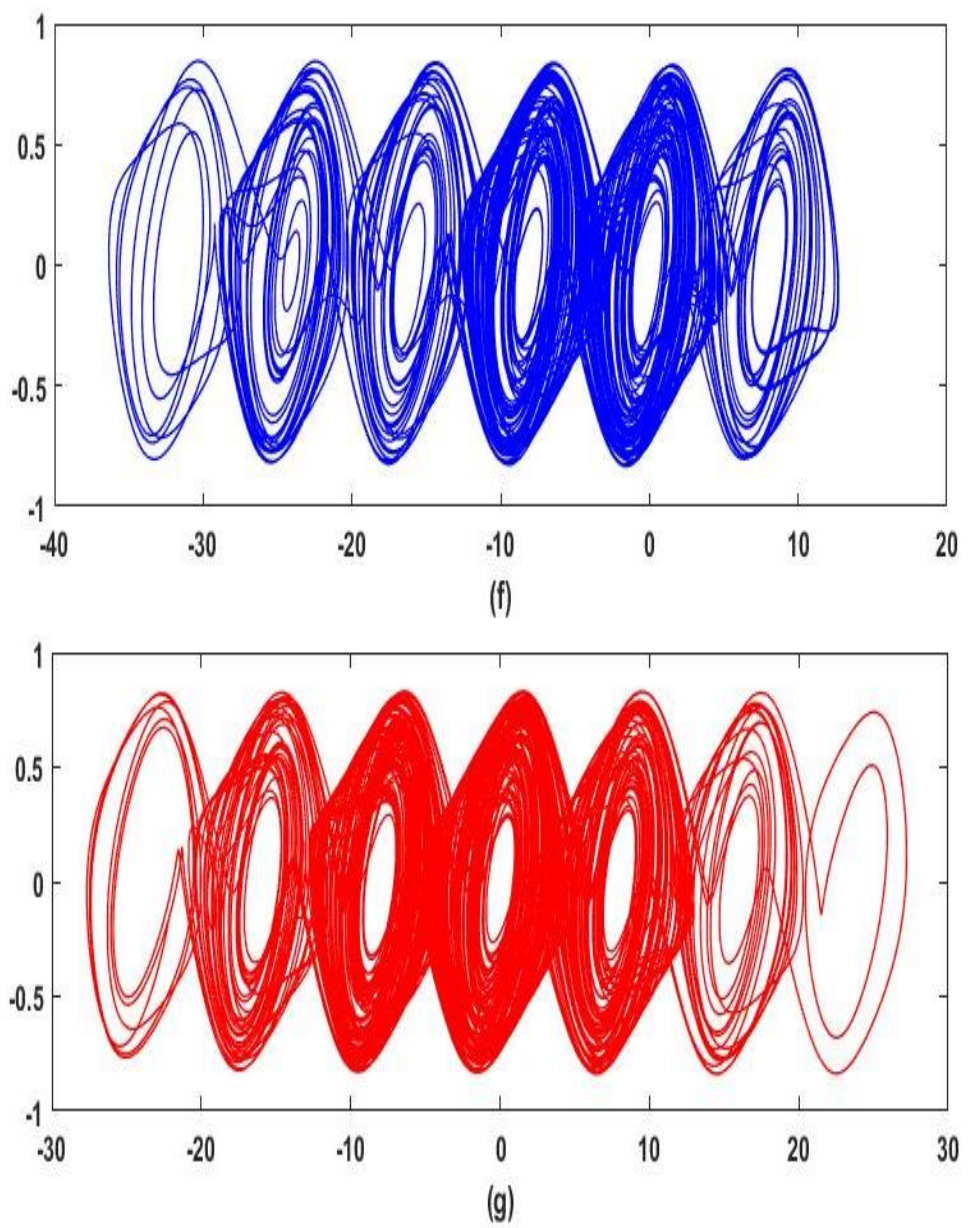

Figure 4. (Continued) 


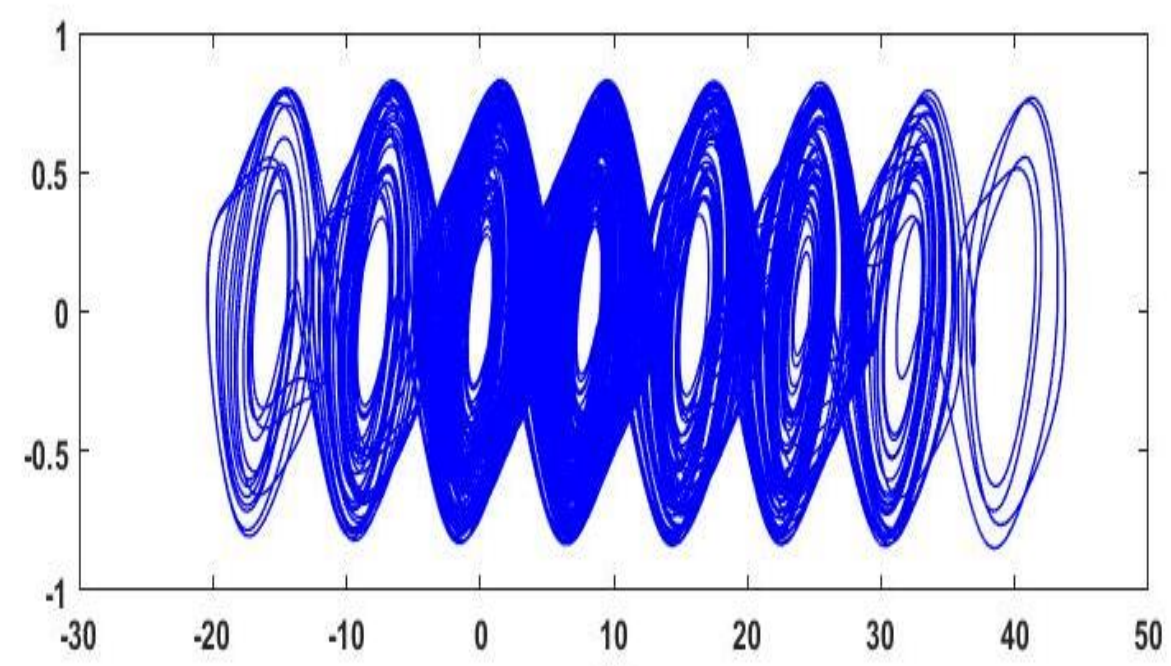

(h)

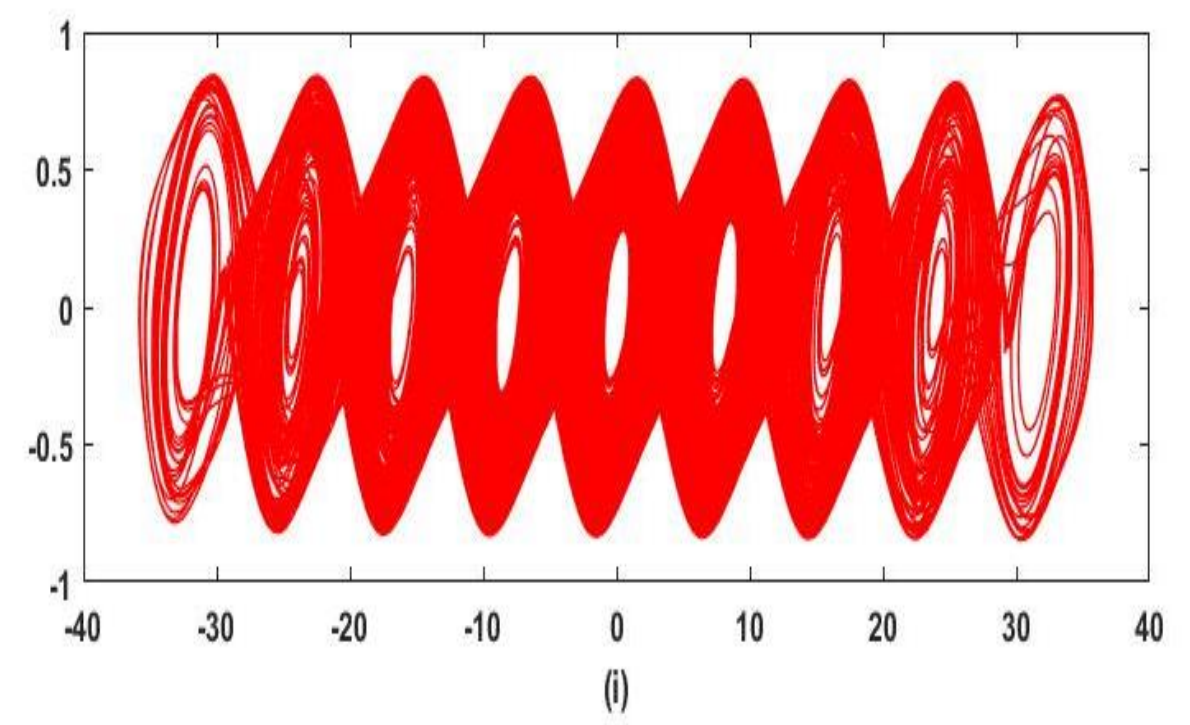

Figure 4. (Continued) 

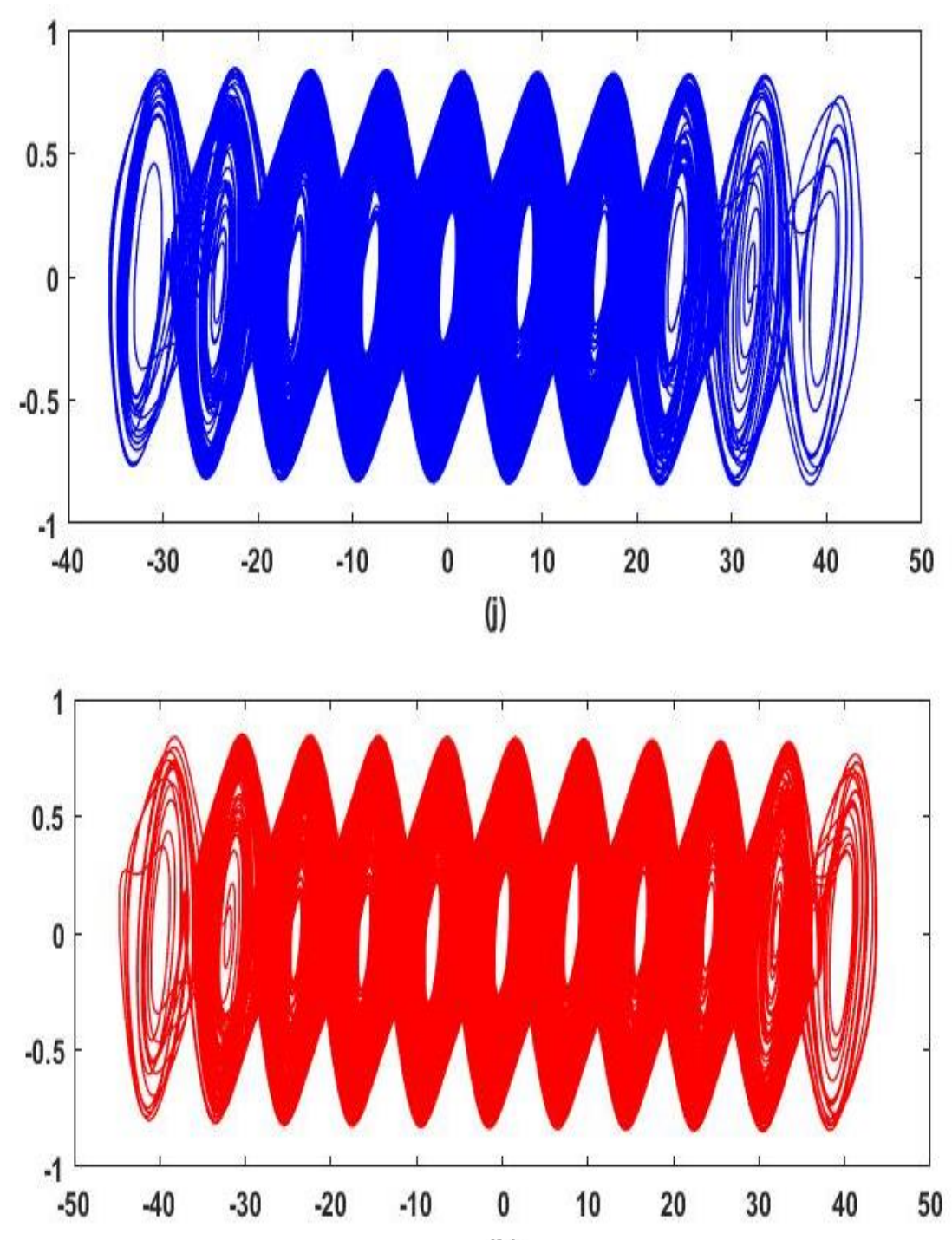

(k)

Figure 4. The Increasing Number of Spirals for the Same Values of $\varepsilon=0.9953$ and Various Values of $t_{\text {stepmax }}$. (a) 1-Spirals for $t_{\text {stepmax }}=144$, (b) 2-Spirals for $t_{\text {stepmax }}=2050$, (c) 3-Spirals for $t_{\text {stepmax }}=2070$, (d) 4-Spirals for $t_{\text {stepmax }}$ $=2700$, (e) 5-Spirals for $t_{\text {stepmax }}=6090$, (f) 6-Spirals for $t_{\text {stepmax }}=6700,(\mathrm{~g}) 7$-Spirals for $t_{\text {stepmax }}=9590$, (h) 8Spirals for $t_{\text {stepmax }}=4000$, (i) 9-Spirals for $t_{\text {stepmax }}=70,000$, (j) 10-Spirals for $t_{\text {stepmax }}=88,210$, (k) 11-Spirals for $t_{\text {stepmax }}=200,000$

In order to highlight the efficiency of this integration duration based method, we repeat the same procedure for all values of $\varepsilon$ of Table 2, we are going to observe the change of scroll number and we resume our results in Table 4 and Figure 5. 
Table 4. Values of $t_{\text {stepmax }}$ for $\varepsilon=0.80$ to $\varepsilon=0.9994$ and $c=12$

\begin{tabular}{|c|c|c|c|c|c|c|}
\hline \multirow{2}{*}{$\varepsilon=0.80$} & $t_{\text {stepmax }}$ & 50,000 & & & & \\
\hline & $\mathrm{Nb}$ of spirals & 1 & & & & \\
\hline \multirow{2}{*}{$\varepsilon=0.86$} & $t_{\text {stepmax }}$ & 7200 & 19,000 & 41,430 & & \\
\hline & $\mathrm{Nb}$ of spirals & 1 & 2 & 3 & & \\
\hline \multirow{2}{*}{$\varepsilon=0.97885$} & $t_{\text {stepmax }}$ & 195 & 680 & 20,000 & 90,000 & 100,000 \\
\hline & $\mathrm{Nb}$ of spirals & 1 & 2 & 3 & 4 & 5 \\
\hline \multirow{4}{*}{$\varepsilon=0.989$} & $t_{\text {stepmax }}$ & 95 & 350 & 1000 & 1380 & 6000 \\
\hline & $\mathrm{Nb}$ of spirals & 1 & 2 & 3 & 4 & 5 \\
\hline & $t_{\text {stepmax }}$ & 80,000 & 90,110 & & & \\
\hline & $\mathrm{Nb}$ of spirals & 6 & 7 & & & \\
\hline \multirow{4}{*}{$\varepsilon=0.993$} & $t_{\text {stepmax }}$ & 85 & 2260 & 2300 & 2340 & 9310 \\
\hline & $\mathrm{Nb}$ of spirals & 1 & 2 & 3 & 4 & 5 \\
\hline & $t_{\text {stepmax }}$ & 9410 & 36,090 & 36,322 & 90,000 & \\
\hline & $\mathrm{Nb}$ of spirals & 6 & 7 & 8 & 9 & \\
\hline \multirow{6}{*}{$\varepsilon=0.9953$} & $t_{\text {stepmax }}$ & 144 & 2050 & 2070 & 2700 & 6090 \\
\hline & $\mathrm{Nb}$ of spirals & 1 & 2 & 3 & 4 & 5 \\
\hline & $t_{\text {stepmax }}$ & 6700 & 9590 & 14,000 & 60,000 & 88,210 \\
\hline & $\mathrm{Nb}$ of spirals & 6 & 7 & 8 & 9 & 10 \\
\hline & $t_{\text {stepmax }}$ & 200,000 & & & & \\
\hline & $\mathrm{Nb}$ of spirals & 11 & & & & \\
\hline \multirow{6}{*}{$\varepsilon=0.9994$} & $t_{\text {stepmax }}$ & 91 & 910 & 1435 & 2250 & 3590 \\
\hline & $\mathrm{Nb}$ of spirals & 1 & 2 & 3 & 4 & 5 \\
\hline & $t_{\text {stepmax }}$ & 4450 & 5610 & 8070 & 8773 & 10,125 \\
\hline & $\mathrm{Nb}$ of spirals & 6 & 7 & 8 & 9 & 10 \\
\hline & $t_{\text {stepmax }}$ & 15,000 & 20,000 & 70,000 & & \\
\hline & $\mathrm{Nb}$ of spirals & 11 & 12 & 13 & & \\
\hline
\end{tabular}




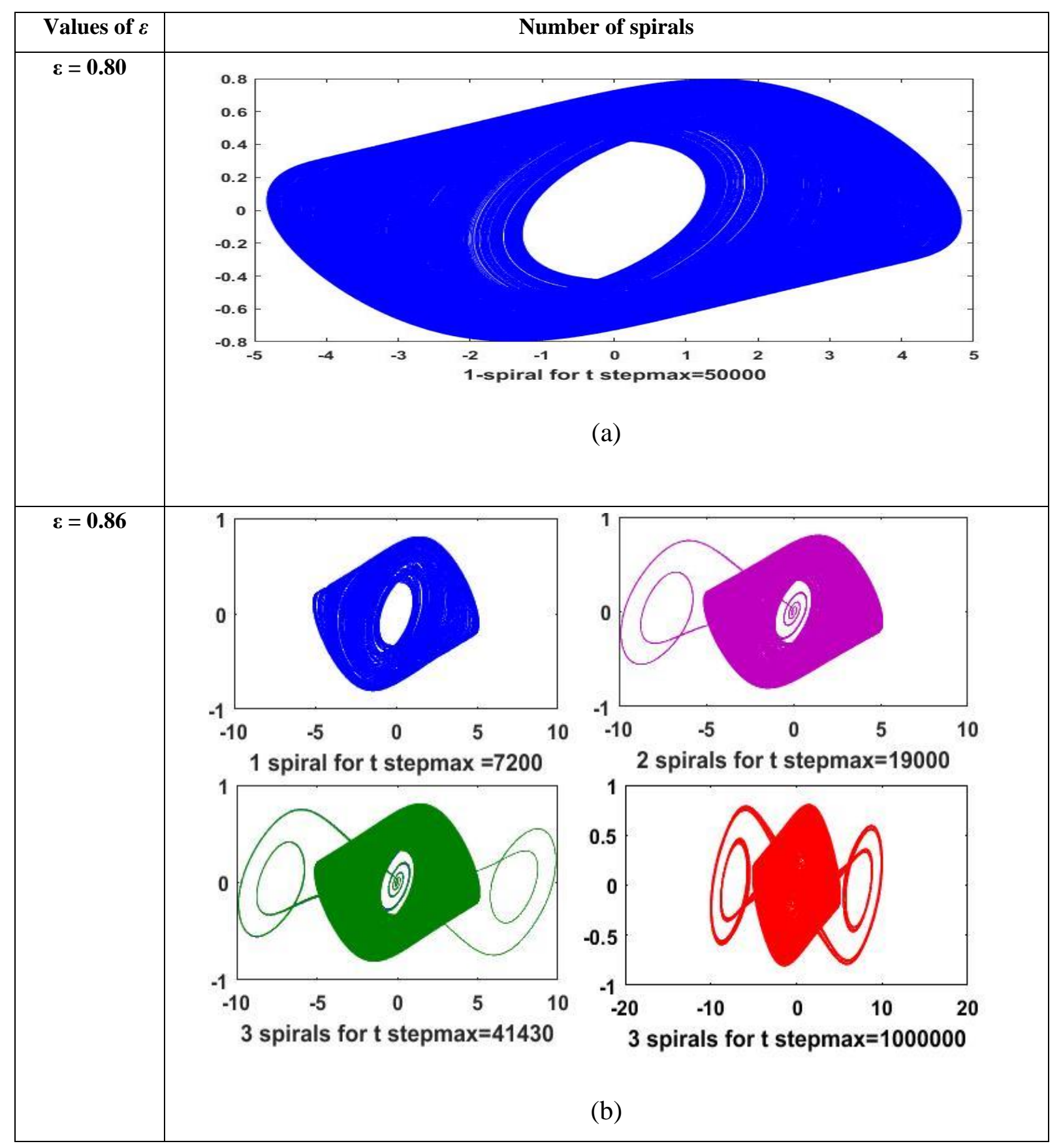




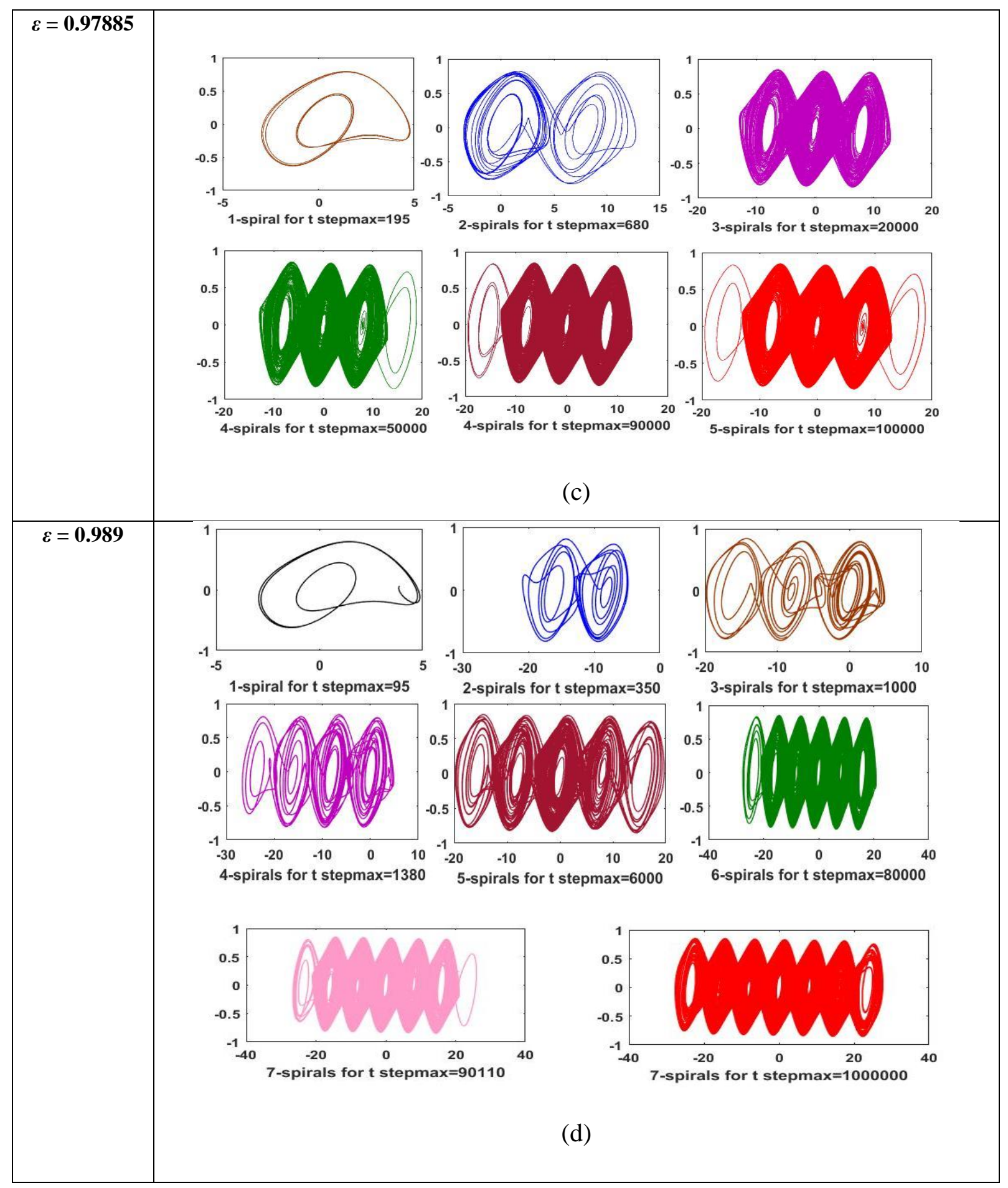




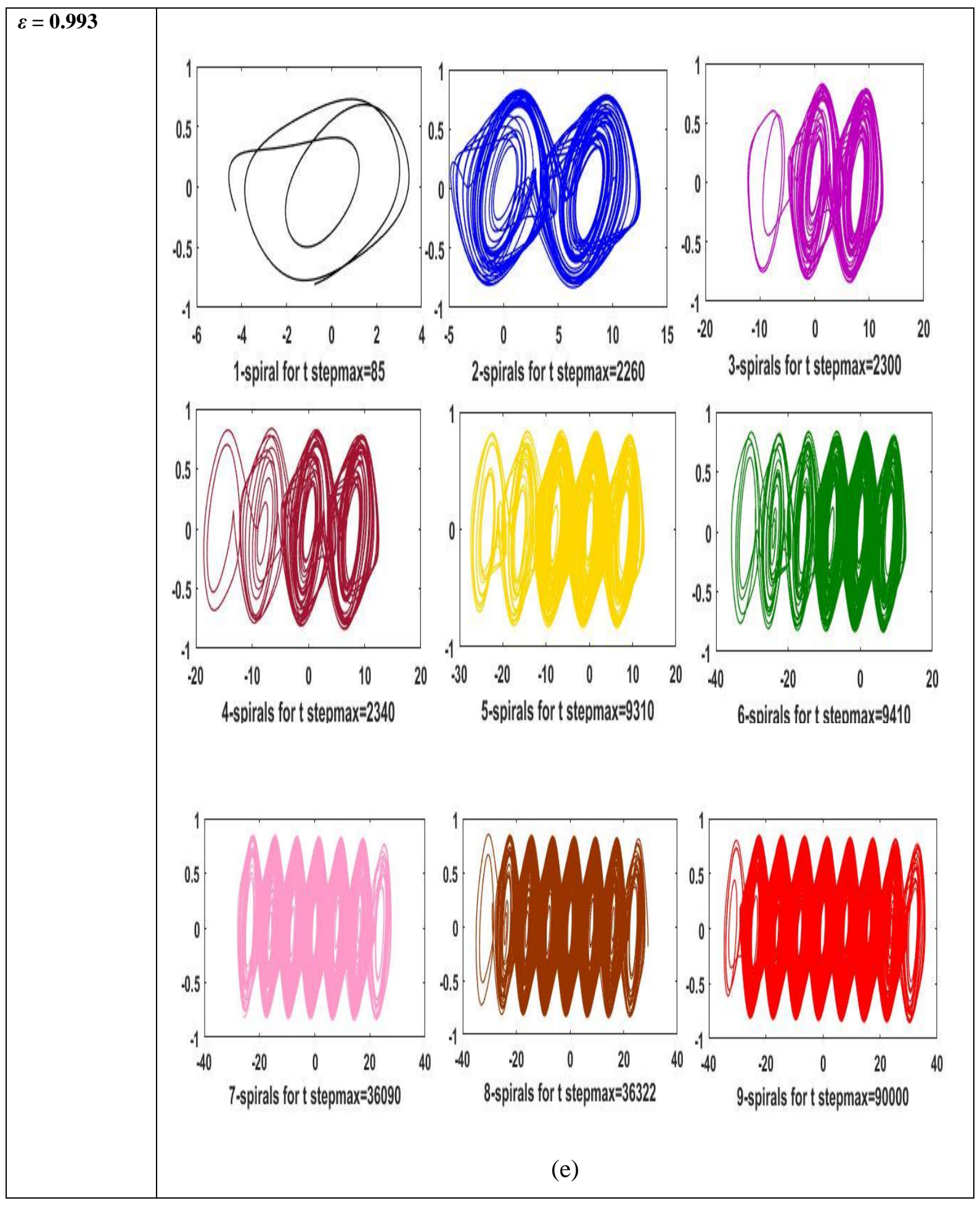




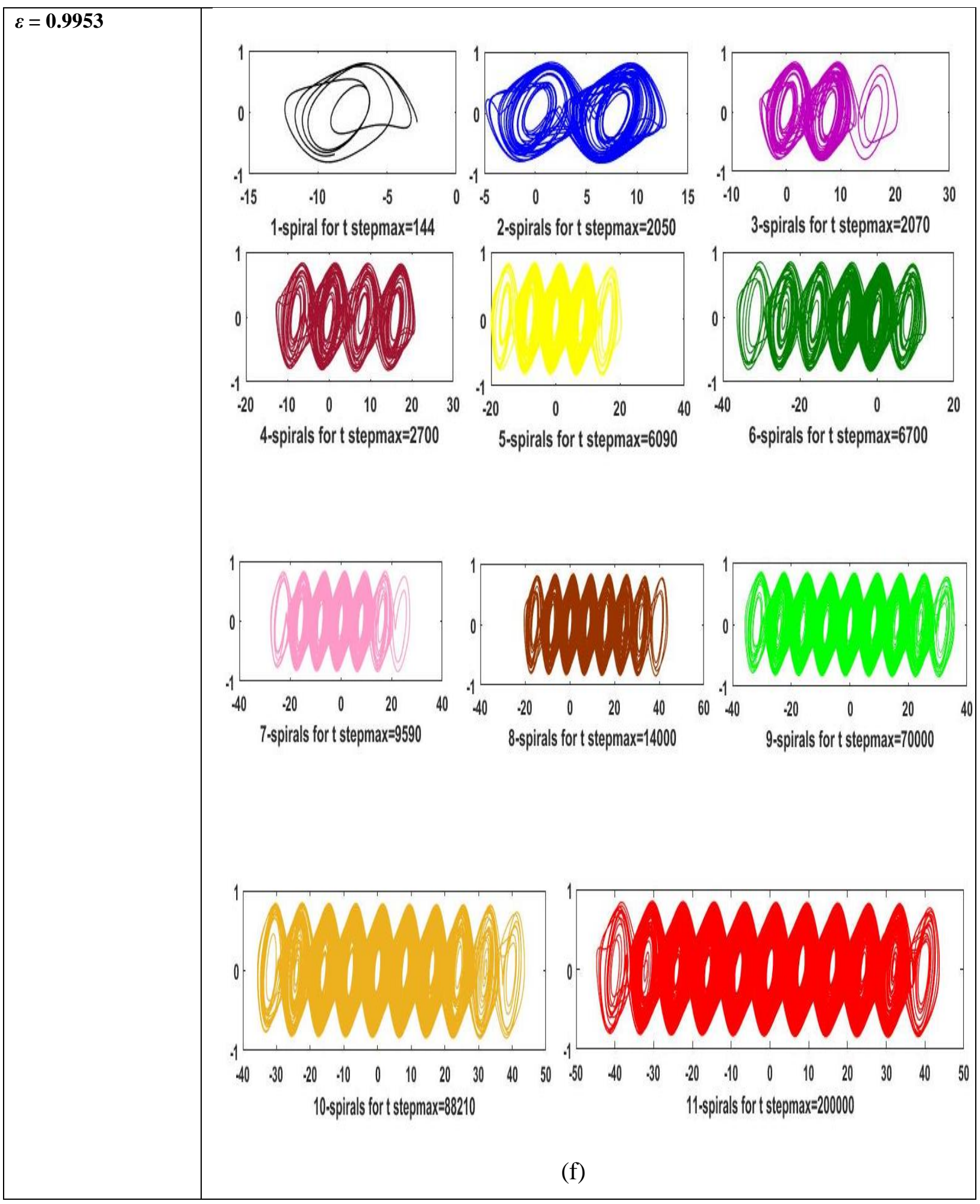




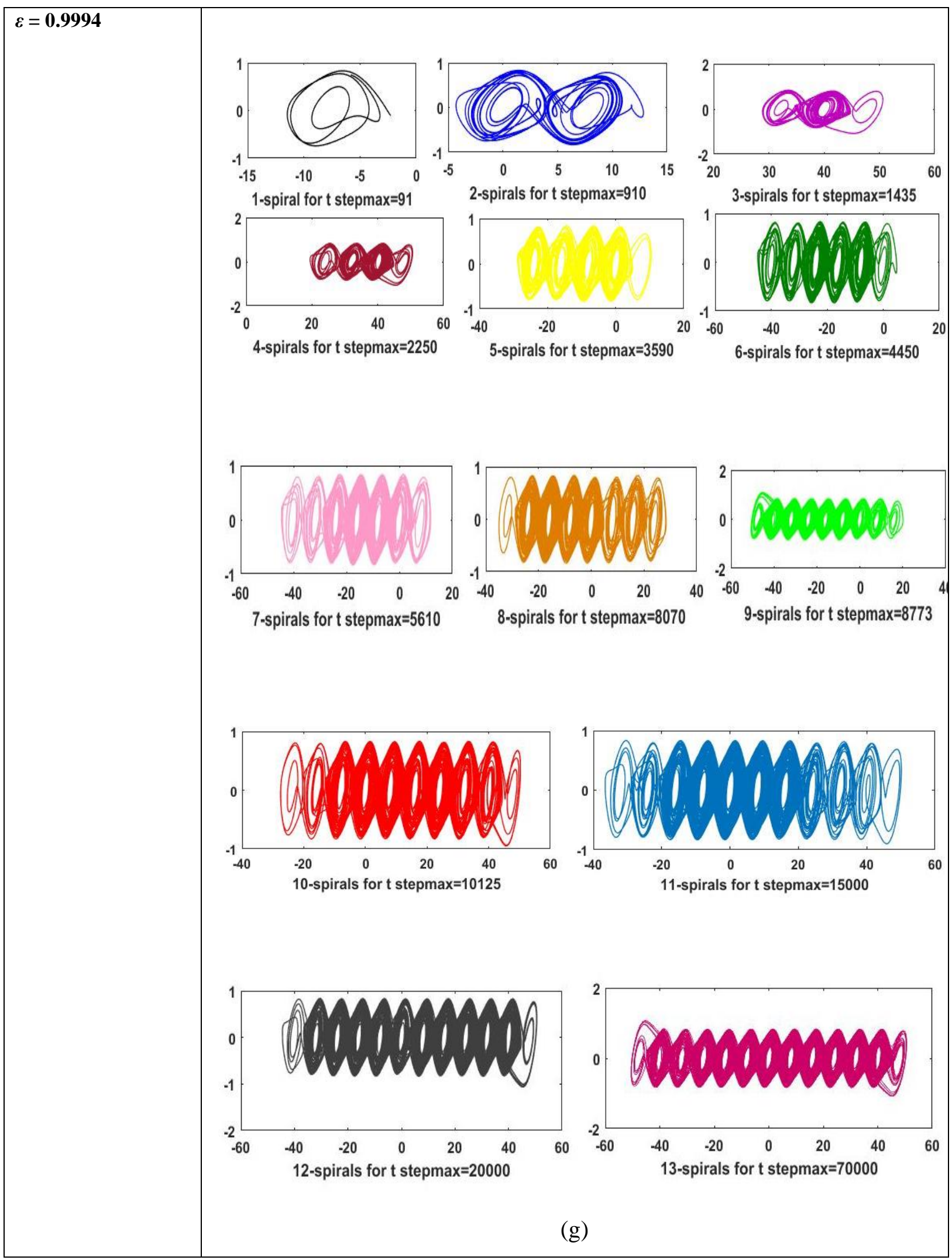

Figure 5. The Increasing Number of Spirals for the Same Values of $\varepsilon$ and Various Values of $t_{\text {stepmax }}$ 


\section{CONCLUSION}

In this article, we have introduced a novel method for unveiling hidden patterns of even number of spirals in the multi-spiral Chua Chaotic attractor. This method is based on the duration of integration of this system and allows displaying every pattern containing from 1 to $\mathrm{c}+1$ spirals of this attractor. After having given the equation of the multi-spiral Chua Chaotic attractor, the MLC method for uncovering hidden bifurcations is explained again and a numerical example of a route of bifurcation is given. In the chosen example, it is shown that the attractors found along this hidden bifurcation route display an odd number of spirals. With the novel method, during the integration process, before reaching the asymptotical attractor which possesses and odd the number of spirals, the number of spirals increases one by one until it reach the maximum number corresponding to the value fixed by $\varepsilon$, unveiling both patterns of even and odd number of spirals.

\section{APPENDIX}

\section{ANALYTICAL-NUMERICAL METHOD FOR HIDDEN ATTRACTOR LOCALISATION}

Ten years ago, Kuznetsov and Leonov ${ }^{[25,26]}$ proposed an effective method for the numerical localisation of hidden attractors in multi-dimensional dynamical systems.

Their method is based on homotopy and numerical continuation. They construct a sequence of similar systems such that for the first (starting) system the initial data for numerical computation of an oscillating solution (starting oscillation) can be obtained analytically. Then the transformation of this starting solution is tracked numerically in passing from one system to another. This proposed approach is generalised in the work [23] to the systems of the form

$$
\frac{d X}{d t}=M X+\underline{H}(X)
$$

where $M$ is a constant $n \times n$ matrix, $\underline{H}(X)$ is a continuous vector-function and $\underline{H}(0)=0$.

They consider a system having one scalar non-linearity, which takes the following form:

$$
\frac{d X}{d t}=M X+q H\left(r^{T} X\right), X \in \mathbb{R}^{n}
$$

Here $q, r$ are constant $n$-dimensional vectors, ${ }^{T}$ is the transposition operation, $H(\sigma)$ is a continuous piecewise differentiable scalar function satisfying the condition $H(0)=0$.

They then define a coefficient of harmonic linearisation $\chi$ in such a way that the matrix

$$
M_{0}=M+\chi q r^{T}
$$

has a pair of purely imaginary eigenvalues $\pm \omega_{0},\left(\omega_{0}>0\right)$ and the rest of its eigenvalues have negative real parts. They assume that such $\chi$ exists and they can rewrite the System (II) as

$$
\frac{d X}{d t}=M_{0} X+q g\left(r^{T} X\right)
$$


where $g(\tau)=H(\tau)-\chi \tau$.

After that, they introduce a finite sequence of functions $g^{0}(\tau), g^{1}(\tau), \ldots, g^{m}(\tau)$ such that the graph of the functions $g^{j}(\tau)$ and $g^{j+1}(\tau),(j=0, \ldots, m-1)$ differs slightly from one to another, where the function $g^{0}(\tau)$ is the small and $g^{m}(\tau)=g(\tau)$. Using the smallness of function, they can apply the method of harmonic linearisation (describing function method) for the system

$$
\frac{d X}{d t}=M_{0} X+q g^{0}\left(r^{T} X\right)
$$

in order to determine the non-trivial stable periodic solution $X^{0}(t)$. For the localisation of the attractor of the original System (IV), they follow numerically the transformation of this periodic solution (a starting oscillating attractor, not including equilibria, denoted further by $A_{0}$ with increasing $j$ ).

To determine the initial condition $X^{0}(0)$ of the periodic solution, the System (V) can be transformed by a linear non-singular transformation $S(X=S Z)$ to the form

$$
\left\{\begin{array}{l}
\dot{z}_{1}(t)=-\omega_{0} z_{2}(t)+b_{1} g^{0}\left(z_{1}(t)+c_{3}^{T} Z_{3}(t)\right) \\
\dot{z}_{2}(t)=\omega_{0} z_{1}(t)+b_{2} g^{0}\left(z_{1}(t)+c_{3}^{T} Z_{3}(t)\right) \\
\dot{Z}_{3}(t)=A_{3} Z_{3}(t)+B_{3} g^{0}\left(z_{1}(t)+c_{3}^{T} Z_{3}(t)\right)
\end{array}\right.
$$

Here, $z_{1}(t), z_{2}(t)$ are scalar values, $Z_{3}(t)$ is a $(n-2)$ dimensional vector; $B_{3}$ and $c_{3}$ are $(n-2)$ dimensional vectors, $b_{1}$ and $b_{2}$ are real numbers; $A_{3}$ is $(n-2) \times(n-2)$ matrix, whose all of its eigenvalues have negative real parts. Without loss of generality, it can be assumed that for the matrix $A_{3}$ there exists a positive number $d_{1}>0$ such that

$$
Z_{3}^{T}(t)\left(A_{3}+A_{3}^{T}\right) A Z_{3}(t) \leq-2 d_{1}\left|\left(Z_{3}(t)\right)^{2}\right|, \quad \forall Z_{3} \in R^{n-2},
$$

In the scalar case, they define the following describing function $G$ of a real variable $\tau$ and assume the existence of its derivative:

$$
G(\tau)=\int_{0}^{\frac{2 \pi}{\omega_{0}}} g\left(\cos \left(\omega_{0} t\right) \tau\right) \cos \left(\omega_{0} t\right) d t
$$

Theorem 1 [25] If it can found a positive $\tau_{0}$ such that

$$
G\left(\tau_{0}\right)=0,\left.\quad b_{1} \frac{d G(\tau)}{d \tau}\right|_{\tau=\tau_{0}}<0
$$

then for the initial condition of the periodic solution $X^{0}(0)=S\left(z_{1}(0), z_{2}(0), Z_{3}(0)\right)^{T}$.

At the initial step of the algorithm, one has $z_{1}(0)=\tau_{0}+O(\varepsilon), \quad z_{2}(0)=0, \quad Z_{3}(0)=$ $O_{n-2}(\varepsilon)$, where $O_{n-2}(\varepsilon)$ is an $(n-2)$ dimensional vector such that all its components are $O(\varepsilon)$.

For the stability of $X^{0}(t)$ (where stability is defined in the sense that for all solutions with the initial data sufficiently close to $X^{0}(0)$ the modulus of their difference with $X^{0}(t)$ is uniformly bounded for all $t>0$ ), it is sufficient to require the following condition is true: 


$$
\left.b_{1} \frac{d G(\tau)}{d \tau}\right|_{\tau=\tau_{0}}<0
$$

In practice, to determine $\chi$ and $\omega_{0}$, one uses the transfer function $W(m)$ of the System (IV)

$$
W(m)=r(M-m I)^{-1} q
$$

where $m$ is a complex variable. The number $\omega_{0}$ is determined from the equation $\operatorname{Im} W\left(i \omega_{0}\right)=0$ and $\chi$ is calculated then by the formula $\chi=-\operatorname{Re} W\left(i \omega_{0}\right)^{-1}$.

\section{REFERENCES}

[1] Poincaré H. 1885. L'Équilibre d'une masse fluide animée d'un mouvement de rotation. Acta Mathematica, 7(1), pp. 259-380.

[2] Sattinger DH, 1973. Topics in stability and bifurcation theory, Lecture Notes in math, Springer-Verlag, Berlin/New York, p. 309.

[3] Lozi R, 1975. A computing method for bifurcation boughs of nonlinear eigen value problems. Bulletin of the American Mathematical Society, 81(6), pp. 1127-1129.

[4] Marsden JE, McCracken M, 1976. The Hopf bifurcation and its applications. Applied Mathematical Sciences, 19, p. 424.

[5] Lorenz E, 1963. Deterministic non-periodic flow. Journal of Atmospheric Science, 20(2), p. 130-141.

[6] Chua LO, 1992. The genesis of Chua's circuit. Archiv für Elektronik und Ubertragung-stechnik, 46, pp. 250-257.

[7] Duan Z, Wang J, Huang L, 2004. Multiinput and multi-output nonlinear systems interconnected Chua's circuits. International Journal of Bifurcation and Chaos, 14(3), pp. 3065-3081.

[8] Zhong GQ, Ayrom F, 1985. Experimental confirmation of chaos from Chua's circuit. International Journal of Circuit Theory and Applications, 13, pp. 93-98.

[9] Matsumoto T, 1984. A chaotic attractor from Chua's Circuit. IEEE Transaction on Circuits and Systems, 31, pp. 1055-1558.

[10] Chua LO, Komuro M, Matsumoto T, 1986. The double scroll family. IEEE Transactions on Circuits and Systems, 33, pp. 1072-1118.

[11] Lozi R, Ushiki S, 1993. The theory of confinors in Chua's circuit: accurate analysis of bifurcations and attractors. International Journal of Bifurcation and Chaos, 3(2), pp. 333-361.

[12] Boughaba S, Lozi R, 2000. Fitting trapping regions for Chua's attractor-a novel method based on isochronic lines. International Journal of Bifurcation and Chaos, 10(1), pp. 205-225.

[13] Khibnik AI, Roose D, Chua LO, 1993. On periodic orbits and homoclinic bifurcations in Chua's circuit with a smooth nonlinearity. International Journal of Bifurcation and Chaos, 3, pp. 363-384.

[14] Shilnikov LP, 1994. Chua's circuit: rigorous results and future problems. International Journal of Bifurcation and Chaos, 4, pp. 489-519.

[15] Huang AS, Pivka L, Wu CW, Franz M, 1996. Chua's equation with cubic nonlinearity. International Journal of Bifurcation and Chaos, 12A, pp. 2175-2222.

[16] Hirsch MW, Smale S, Devaney RL, 2003. Differential equations, dynamical systems and an introduction to chaos. Second edition. Elsevier, Academic Press, Amsterdam.

[17] Suykens JAK, Vandewalle J, 1991. Quasilinear approach to nonlinear systems and the design of ndouble scroll $(n=1 ; 2 ; 3 ; 4 ; \ldots)$. Proceedings of the IEEE. Institute of Electrical and Electronics Engineers, 138(5), pp. 595-603.

[18] Arena P, Baglio S, Fortuna L, Manganaro G, 1996. Generation of $n$-double scrolls via cellular neural networks. International Journal of Circuit Theory and Applications, 24(3), pp. 241-252.

[19] Suykens JAK, Huang A, Chua LO, 1997. A family of n-scroll attractors from a generalized Chua's circuit. International Journal of Electronics and Communications, 51(3), pp. 131-138.

[20] Yalçin ME, Suykens JAK, Vandewalle J, 2000. Experimental confirmation of 3-and 5-scroll theory attractors from a generalized Chua's circuit. IEEE Transaction on Circuits and Systems-Part I Fundamental and Applications, 47(3), pp. 425-429. 
[21] Tang KS, Zhong GQ, Chen G, Man KF, 2001. Generation of n-scroll attractors via sine function. IEEE Transactions on Circuits and Systems, 48(11), pp. 1369-1372.

[22] Leonov GA, Kuznetsov NV, Vagaitsev VI, 2011. Localization of hidden Chua's attractors. Physics Letters A, 375, pp. 2230-2233.

[23] Leonov GA, Kuznetsov NV, 2011. Analytical-numerical methods for investigation of hidden oscillations in nonlinear control systems. Preprints of the 18th IFAC World Congress, Milano, Italy, 2, pp. 2494-2505.

[24] Leonov GA, 2009. On harmonic linearization method. Automation and Remote Control, 5, pp. 65-75.

[25] Leonov GA, Vagaitsev VI, Kuznetsov NV, 2010. Algorithm for localizing Chua attractors based on the harmonic linearization method. Doklady Mathematics D, 82, pp. 663-666.

[26] Leonov GA, Kuznetsov NV, Vagaitsev VI, 2012. Hidden attractor in smooth Chua systems. Physica D, 241, pp. 1482-1486.

[27] Menacer T, Lozi R, Chua L, 2016. Hidden bifurcations in the multispiral Chua attractor. International Journal of Bifurcation and Chaos, 26(14), p. 1630039 (26 pages).

[28] Madan RN, editor, 1993. Chua's circuit: a paradigm for chaos. World Scientific Series on Nonlinear Science Series B, 1, p. 1043.

[29] Lur'e AI, 1951. Certain nonlinear problems in the theory of automatic control. Gostekhizat, Moscow, Leningrad. Translated into English, H.M. Stationery, 1957. 